\title{
MINI-SYMPOSIUM
}

\section{Pathology of coronary microembolisation and no reflow}

\author{
E Falk, L Thuesen
}

Heart 2003;89:983-985

C oronary atherosclerosis is the underlying cause of nearly all cases of ischaemic heart disease, and superimposed thrombosis is the cause of the great majority of acute coronary syndromes. ${ }^{12}$ The pathogenesis of peripheral arterial disease and, to a great extent, ischaemic stroke is similar. Thus, "atherothrombosis" is the leading cause of severe disability and cardiovascular death.

\section{ATHEROTHROMBOTIC BURDEN}

In general, atherothrombotic plaques responsible for acute coronary syndromes are larger (hidden in positively remodelled arteries) and softer (contain more lipid, inflammation and thrombus and less calcification) than angina producing lesions. ${ }^{12}$ Plaques in aortocoronary saphenous vein grafts (SVG) are, in general, extraordinarily bulky, friable, and thrombus-rich, regardless of clinical presentation. ${ }^{3}$ Recent observations indicate that the atherothrombotic burden is a major determinant of coronary microembolisation, particularly when plaques are crushed and fragmented mechanically during percutaneous coronary interventions (PCI).

\section{Saphenous vein graft lesions}

Atherogenesis is notably accelerated in SVGs, and fatal atherothrombosis may develop within a few years after grafting (fig lA). ${ }^{3}$ Compared to atherothrombosis in native coronary arteries, plaques in SVGs are generally much larger and contain more lipid, inflammation (foam cells), and thrombus and less calcification. ${ }^{3}$ Consequently, the atherothrombotic burden is larger and the plaques are much more friable (vulnerable), which explains the exceptionally high risk of distal embolisation, particularly when old SVGs are manipulated by surgeons' hands or cardiologists' devices.

\section{Coronary thrombosis}

Approximately $75 \%$ of coronary thrombi are precipitated by rupture of a soft and "vulnerable" plaque, ${ }^{12}$ and the same mechanism underlies atherothrombotic occlusion of SVGs. ${ }^{4}$ Platelet aggregation plays a critical role initially during the evolution of a coronary thrombus, but blood stagnation and coagulation contribute significantly to the overall thrombotic burden once the platelet-rich thrombus occludes the lumen totally (fig 1B). Lack of side branches favour blood stagnation and an enormous amount of thrombus may develop in occluded large calibre SVGs. Furthermore, thrombi in SVGs often persist and organise slowly, if at all.

\section{ATHEROTHROMBOTIC MICROEMBOLISATION}

Lipid-rich and inflamed plaques are vulnerable to rupture. By rupturing, the soft atheromatous gruel is suddenly exposed to the flowing blood which increases the risk of both local thrombosis and distal embolisation of atherothrombotic material. ${ }^{5}$ The latter phenomenon, known as athero- or cholesterol embolisation, has been described in aorta, carotid, coronary, and other arteries. Superimposed thrombosis may seal a ruptured plaque and prevent distal atheroembolisation, but thrombolysis may re-expose the soft gruel and thus revive the risk of atheroembolisation.

\section{Spontaneous coronary microembolisation}

A ruptured plaque with a superimposed non-occlusive thrombosis can, in principle, shower and obstruct the microcirculation with soft plaque material (atheroembolisation) and/or thrombotic material (thromboembolisation). Postmortem studies of patients who died after a thrombus mediated heart attack have revealed thromboemboli and, more rarely, atheroemboli impacted downstream in small intramyocardial arteries in a high proportion of cases..$^{6-9}$ The overall microembolic burden is unknown but is probably relatively low compared to what may happen after PCI. Troponin elevations in acute coronary syndromes without ST elevation indicate (micro)infarction of thrombotic, but not necessarily thromboembolic, origin..$^{5}$ A dynamic atherothrombotic lesion in an epicardial artery may cause subendocardial ischaemia and (micro) necrosis by reducing the blood flow subcritically and/or temporarily without implicating microembolisation. The marginal, if any, protective effect of platelet glycoprotein IIb/IIIa inhibition in acute coronary syndromes without ST elevation treated conservatively (no PCI) could indicate that spontaneous platelet mediated microembolisation plays no major role in this syndrome. ${ }^{10}$ Even troponin positive patients did not receive any benefit from potent platelet inhibition in the large GUSTO IV-ACS trial. ${ }^{10}$

\section{latrogenic coronary microembolisation}

In myocardial infarction with ST elevation, fibrinolytic treatment alone is capable of promoting distal embolisation, but mechanical crushing and fragmentation of the culprit lesion during PCI has emerged as the major cause of coronary microembolisation. ${ }^{5}$ The risk of PCI mediated microembolisation depends on the atherothrombotic burden and the invasiveness of the procedure. ${ }^{11}$ Consequently, it is relatively common in two clinical settings: PCI in stenotic SVGs (bulky and friable plaques, fig $1 \mathrm{~A}$ ) and in acute myocardial infarction (soft plaques + thrombosis, fig 1B); atherectomy and stenting cause more plaque fragmentation and distal embolisation than balloon angioplasty. ${ }^{11}$ Thus, despite otherwise successful recanalisation, PCI induced distal microembolisation and microvascular obstruction may lead to inadequate myocardial perfusion, the so-called "no reflow" phenomenon.

\section{NO/SLOW REFLOW}

The pathogenesis of no/slow reflow after PCI in atherothrombotic heart disease differs significantly from the "classical" no reflow phenomenon seen after temporary occlusion of normal coronary arteries in animals. ${ }^{12}$

\section{Classical no reflow in animals}

After coronary occlusion in dogs, myocytes begin to die in the subendocardial myocardium after $\sim 20$ minutes, and ischaemic

Abbreviations: GUSTO IV-ACS, global use of strategies to open occluded coronary arteries IV - acute coronary syndromes; $\mathrm{PCl}$, percutaneous coronary intervention; SVG, saphenous vein graft; 

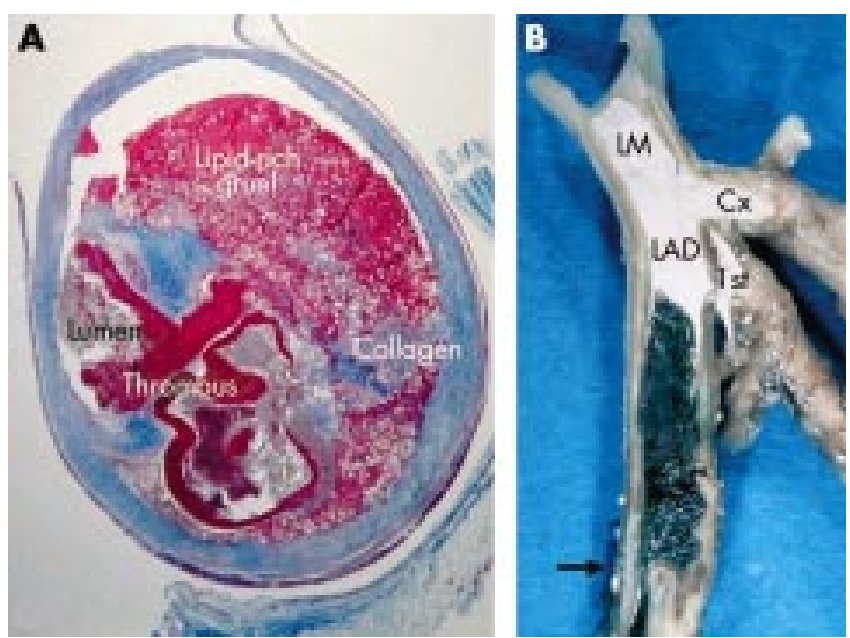

Figure 1 Coronary microembolisation and no reflow is particularly common in two clinical settings; old stenotic saphenous vein grafts ( $A$, high plaque burden) and acute coronary occlusion ( $B$, high thrombotic burden). Where does all this atherothrombotic materia go during percutaneous coronary intervention? (A) Cross-section of a four year old saphenous vein graft, revealing a bulky and lipid-rich (friable) plaque with ruptured surface and superimposed thrombosis (trichrome stain). (B) Left coronary artery opened longitudinally, revealing a dark red stagnation thrombus (erythrocyte-rich) between the first diagonal branch (1 st) and the occlusive white thrombus (platelet-rich) formed on top of a ruptured plaque (arrow). White contrast medium injected postmortem is filling the lumen proximal to the thrombus. $L M$, left main; $L A D$, left anterior descending artery; $C x$, circumflex artery.

cell death progresses from the subendocardium to the subepicardium as a wavefront in a time dependent fashion. ${ }^{12}$ About six hours of ischaemia are required to complete the wavefront of necrosis. Although myocardial necrosis averaging 28\% of the vascular bed has developed after 40 minutes of ischaemia, myocardial reperfusion is still homogeneous without any defect if coronary flow is restored. However, if coronary flow is not restored until after 90 minutes of ischaemia, myocardial perfusion defects (no reflow) are now present in myocardium that was necrotic at an earlier time point, first in the subendocardial zone. ${ }^{12}$ No reflow, or more correct "no reperfusion", appears to be confined to myocardium that is already necrotic and thus follows necrosis and not vice versa. To date, no study of "pure" coronary occlusion (that is, without microembolisation) have demonstrated myocardial no reperfusion preceding myocardial necrosis. ${ }^{12}$ The no reperfusion areas enlarge both with the degree and duration of ischaemia and with the duration of reperfusion (combined ischaemia and reperfusion injury). ${ }^{12} 13$

The inability to reperfuse necrotic myocardium is caused by progressive microvascular occlusion. Many different obstructive mechanisms have been proposed such as endothelial swelling, neutrophil plugging, vascular "squeezing" by ischaemic contracture (intracellular calcium overload), or compression from the surrounding necrotic and swollen myocytes. ${ }^{12}$ Microvessels plugged by platelets and fibrin are also seen, but microembolisation does not occur and fibrinolytic treatment is ineffective in this model.

\section{No reflow after $\mathrm{PCl}$ in myocardial infarction}

Unlike animal models of coronary occlusion, the clinical setting involves an atherothrombotic occlusion with its innate risk of distal embolisation when crushed or fragmented mechanically. Thus, coronary no/slow reflow and myocardial hypoperfusion after otherwise successful recanalisation of infarct related arteries may involve more than just classical no reperfusion confined to myocardium that is already dead. No/slow reflow may also result from PCI induced microvascular obstruction caused by distal microembolisation and/or microvascular spasm. ${ }^{56}$ Because microemboli necessarily stream preferentially to well perfused and viable myocardium, microembolisation kills potentially salvageable myocardium. Thus, the vital question is, of course: how much of the coronary no/slow reflow and myocardial hypoperfusion seen after primary PCI reflects the classical no reflow phenomenon caused by necrosis, and how much reflects PCI induced distal microembolisation (and microvascular spasm?) causing more necrosis? The (athero)thrombotic burden may prove to be critical, indicated by the beneficial effect of platelet glycoprotein IIb/IIIa receptor inhibition before stenting. ${ }^{14}$ Whether thrombectomy (before PCI) or distal embolic protection devices (during PCI) will improve myocardial perfusion and clinical outcomes remains to be shown by ongoing clinical trials.

\section{No reflow after $\mathrm{PCl}$ in old SVGs}

PCI of stenotic SVGs is associated with an exceptionally high risk of macroembolisation (angiographic distal cutoff) and no or low flow through myocardium that was perfused normally before PCI. ${ }^{15}$ PCI induced distal microembolisation and/or microvascular spasm are the most obvious explanations (classical no reflow is irrelevant), recently documented by the pronounced reduction in procedure related no reflow and myocardial infarction with the use of a distal embolic protection device during stenting. ${ }^{15}$ The lack of a consistent benefit with the use of potent antiplatelet agents during PCI in SVGs indicates that embolised atheromatous debris rather than platelet mediated thromboembolism is responsible for the detrimental effects associated with stenting of stenotic SVG lesions. ${ }^{15} 16$

\section{Authors' affiliations}

E Falk, L Thuesen, Department of Cardiology, Aarhus University Hospital (Skejby), Aarhus, Denmark

Correspondence to: Dr Professor Erling Falk, Department of Cardiology, Aarhus University Hospital (Skejby), DK-8200 Aarhus N, Denmark; Erling.Falk@iekf.au.dk

\section{REFERENCES}

1 Davies MJ. The pathophysiology of acute coronary syndromes [review] Heart 2000;83:361-6

2 Falk E. Stable versus unstable atherosclerosis: clinical aspects [review]. Am Heart J 1999;138(5 Pt 2):S42 1-5.

3 Motwani JG, Topol EJ. Aortocoronary saphenous vein graft disease: pathogenesis, predisposition, and prevention [review]. Circulation 1998:97:916-31.

4 Falk E. Fatal atherosclerosis developed in less than 4 years in an aortocoronary vein graft in a hyperlipidemic patient. Acta Pathol Microbiol Immunol Scand [A] 1984;92:73-5.

5 Topol EJ, Yadav JS. Recognition of the importance of embolization in atherosclerotic vascular disease [review]. Circulation 2000;101:570-80.

6 Heusch G, Schulz R, Baumgart D et al. Coronary microembolization [review]. Prog Cardiovasc Dis 2001;44:217-30.

7 Falk E. Unstable angina with fatal outcome: dynamic coronary thrombosis leading to infarction and/or sudden death. Autopsy evidence of recurrent mural thrombosis with peripheral embolization culminating in total vascular occlusion. Circulation 1985:71:699-708.

8 Davies MJ, Thomas AC, Knapman PA, et al. Intramyocardial platelet aggregation in patients with unstable angina suffering sudden ischemic cardiac death. Circulation 1986;73:418-27.

9 Frink RJ, Rooney PA, Trowbridge JO, et al. Coronary thrombosis and platelet/fibrin microemboli in death associated with acute myocardial infarction. Br Heart J 1988;59:196-200.

10 Roffi M, Chew DP, Mukherjee D, et al. Platelet glycoprotein Ilb/Illa inhibition in acute coronary syndromes. Gradient of benefit related to the revascularization strategy. Eur Heart J 2002;23:1441-8.

11 Mehran R, Dangas G, Mintz GS, et al. Atherosclerotic plaque burden and CK-MB enzyme elevation after coronary interventions: intravascular ultrasound study of 2256 patients. Circulation 2000;101:604-10.

12 Reffelmann T, Kloner RA. The "no-reflow" phenomenon: basic science and clinical correlates [review]. Heart 2002;87:162-8.

13 Reffelmann T, Kloner RA. Microvascular reperfusion injury: rapid expansion of anatomic no reflow during reperfusion in the rabbit. Am J Physiol Heart Circ Physiol 2002;283:H1099-107.

14 Montalescot G. Defining standards of care for facilitated and direct percutaneous coronary intervention [review]. Clin Cardiol 2002;25(suppl l), I-27-32 
15 Baim DS, Wahr D, George B, et al for the Saphenous vein graft Angioplasty Free of Emboli Randomized (SAFER) Trial Investigators. Randomized trial of a distal embolic protection device during percutaneous intervention of saphenous vein aorto-coronary bypass grafts. Circulation 2002;105:1285-90.
16 Roffi M, Mukherjee D, Chew DP, et al. Lack of benefit from intravenous platelet glycoprotein Ilb/lla receptor inhibition as adjunctive treatment for percutaneous interventions of aortocoronary bypass grafts: a pooled analysis of five randomized clinical trials. Circulation 2002;106: 3063-7.

\section{IMAGES IN CARDIOLOGY}

\section{Long term warfarin associated with bilateral blindness in a patient with atrial fibrillation and macular degeneration}

A ge related macular degeneration (ARMD) is the most common cause of poor vision in later life. It is usually atrophic (dry), though in $10-20 \%$ there is a subretinal neovascular response, usually causing more rapid and serious loss of acuity (wet ARMD). Bleeding from these vessels is common, though rarely severe. The usual outcome in ARMD is loss of central vision, with preserved peripheral vision.

This 82 year old patient suffered a myocardial infarction in 1994, complicated by left ventricular thrombus and atrial fibrillation. She recovered well, and is on digoxin and warfarin. In May 2000 she developed wet ARMD in her right eye which (unusually) progressed to total blindness as a result of massive haemorrhage filling the vitreous. During this time the international normalised ratio (INR) varied between 2.5 and 3.1.

In November 2001 she developed wet ARMD in her left eye, initially with a small haemorrhage under the macula (below left). Vision then dramatically deteriorated, due (again) to

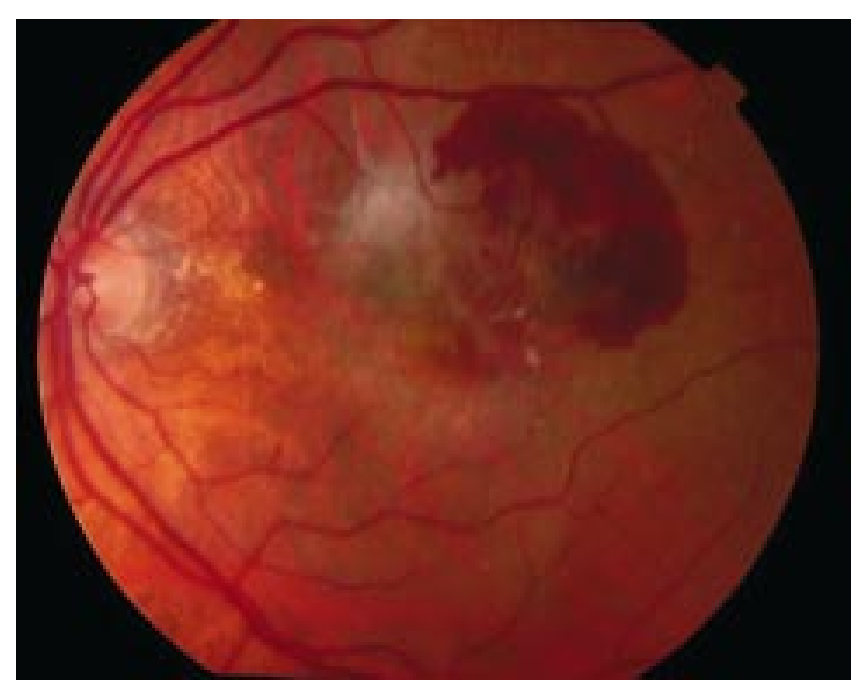

bleeding into the vitreous, and this time the INR had unexpectedly risen to 4.1. Vitrectomy surgery was undertaken to remove all vitreous blood, though blood in the choroid was not accessible. Below right (postoperation) shows a large mass of blood in the choroid, pushing the inferior and superior retina forward, making it out of focus. The patient has now regained some peripheral (navigational) vision.

Atrial fibrillation and ARMD are common co-morbidities in the elderly. We recommend that patients on warfarin who develop wet ARMD should be advised to maintain an INR at the lower end of the recommended range. With second eye involvement consideration should be given to an alternative anticoagulation strategy altogether.

T Ung

$M$ James

R H Gray

roger.gray@tst.nhs.uk

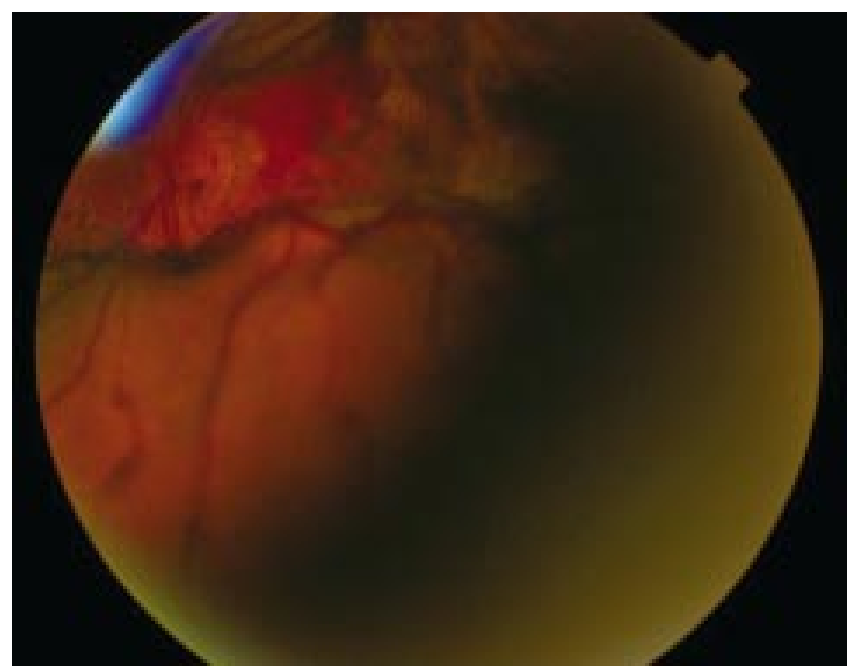

\section{ELECTRONIC PAGES}

\section{eHEART: www.heartinl.com}

$\mathrm{T}$ he following electronic only article is published in conjunction with this issue of Heart.

\section{Emergency coronary stenting of unprotected critical left main coronary artery stenosis in acute myocardial infarction and cardiogenic shock \\ $\mathrm{H}$ McArdle, M Bhandari, J Kovac}

In the setting of acute myocardial infarction (MI) and cardiogenic shock in patients with significant unprotected left main coronary artery (LMCA) disease, treatment options are limited. In this report of a patient presenting in cardiogenic shock secondary to acute MI with critical LMCA stenosis, percutaneous coronary intervention with intra-aortic balloon pump support proved life saving.

(Heart 2003;89:e24) www.heartjnl.com/cgi/content/full/89/ $9 / \mathrm{e} 24$ 